\title{
Densidade básica da madeira de espécies arbóreas de Cerradão no estado de Tocantins
}

\author{
Carlos José da Silva ${ }^{1 *}$, Ailton Teixeira do Vale ${ }^{2}$, Eder Pereira Miguel ${ }^{2}$ \\ ${ }^{1}$ Universidade Federal Rural do Semi-Árido, Departamento de Ciências Vegetais, Av. Francisco Mota, 572, Bairro Costa e Silva, CEP 59.625-900, Mossoró, RN, Brasil \\ ${ }^{2}$ Universidade de Brasília, Secretaria de Pós-Graduação em Ciências Florestais, Campus Universitário Darcy Ribeiro, CEP 70910-900, Brasília, DF, Brasil
}

Termos para
Biomassa
Tronco
Galhos
Cerrado
Index terms:
Savanna
Stems
Branches
Biomass

"Autor correspondente:

carlosbioforest@gmail.com

Histórico do artigo:

Recebido em 18/11/2014

Aprovado em 30/04/2015

Publicado em 30/06/2015

doi: $10.4336 / 2015 . p f b .35 .82 .822$
Resumo - O objetivo desse estudo foi analisar a densidade básica de espécies arbóreas da fitofisionomia Cerradão, no domínio do Cerrado no estado do Tocantins, e estabelecer a relação entre a densidade básica da madeira dos troncos em função da densidade básica da madeira dos galhos. Amostras de 80 indivíduos foram coletadas no tronco (base, meio e topo) e galhos (galho grosso, galho médio e galho fino) para determinação da densidade básica. As espécies apresentaram valor médio de $0,650 \mathrm{~g} \mathrm{~cm}^{-3}$, sendo que para a maioria das espécies (58\%) decresceu da base para o topo do tronco. A densidade básica da madeira entre posições axiais do tronco e a densidade básica entre galhos de diferentes diâmetros apresentaram relação significativa. De acordo com os modelos propostos para estimar a densidade do tronco em função da densidade de galhos, a variável densidade do galho médio apresentou o melhor ajuste na equação. As equações desenvolvidas neste trabalho são adequadas para estimativas de biomassa e estoque de carbono de espécies arbóreas da fitofisionomia Cerradão, sem amostragem destrutiva de indivíduos arbóreos.

\section{Basic wood density tree species of Cerradão physiognomy in the state of Tocantins}

\begin{abstract}
The aim of this study was to analyze the basic density of tree species of Cerradão phytophysiognomy in Cerrado domain in the state of Tocantins, and establish the relationship between the wood basic density of stems and branches. Samples of 80 trees were collected from stems (base, middle and top) and branches (thick branch, medium branch, and twig). The species had an average $0,650 \mathrm{~g} \mathrm{~cm}^{-3}$ of wood basic density. The basic density for most species (58\%) decreased from the bottom to the top of the stem. The basic wood density between axial positions of the stem and the basic density of branches with different diameters showed significant relationship. According to proposed models to estimate stem density using branches density, the medium branch density showed the best adjust in the equation. The developed equations are adequate to estimate biomass and carbon stock of trees from Cerradão physiognomy, without destructive sampling.
\end{abstract}




\section{Introdução}

A grande diversidade de formas de vida vegetal nas florestas tropicais é condicionada pelo clima quente e úmido (Pringle, 1997). O bioma Cerrado, por se situar em uma área de contato com zonas climáticas distintas, apresenta aspecto ecológico único, bem como fisionomias peculiares. A biomassa florestal e a energia potencial contida na mesma estão distribuídas nos compartimentos aéreos e subterrâneos das espécies florestais, em diferentes porcentagens. A caracterização da madeira, pela determinação de sua densidade ou da massa específica, e de sua variação dentro da árvore, tanto na direção radial, da medula para a casca, quanto no sentido base/topo, é fundamental como subsídio ao entendimento de sua qualidade (Oliveira et al., 2005). Valério et al. (2008) relatam que a densidade da madeira é influenciada por vários fatores, entre eles idade, procedência, local de origem, espaçamento, taxa de crescimento entre gêneros e espécies. $\mathrm{O}$ conhecimento desta variabilidade é importante para otimizar a exploração da madeira, assim como para aplicar novas técnicas e métodos para facilitar os levantamentos do potencial madeireiro.

A densidade básica é sempre uma medida da densidade aparente na madeira (Trugilho et al., 1990) e pode ser determinada pela relação entre a massa seca a $0 \%$ de umidade (massa anidra) e o volume verde, ou pela relação com o máximo teor de umidade (Associação Brasileira de Normas Técnicas, 2003). A densidade básica apresenta variações devido a diferenças na estrutura e a presença de substâncias extrativas na madeira, sendo que a estrutura é caracterizada pela quantidade proporcional de diferentes tipos de células (Costa, 2006). A densidade básica é uma das características que melhor expressa as propriedades funcionais da madeira, como foi ressaltado em diversos trabalhos (Sih, 1974; Ribeiro \& Zani Filho, 1993; Bowyer et al., 2003; Muller-Landau, 2004; Preston et al., 2006; Williamson \& Wiemann, 2011; Jati et al., 2014), dentre outros.

O entendimento das variações da densidade da madeira em função das espécies e ambientes é um instrumento importante para a transformação de dados de volume de madeira em biomassa (Chave et al., 2006; Jati et al., 2014). Para esses autores, esses estudos são importantes pois resultam em dados que reforçam o conhecimento da dinâmica dos estoques de carbono terrestres regionalizados, os quais dão suporte científico aos acordos internacionais sobre mudanças climáticas globais. No entanto, este tema é pouco investigado em áreas de savanas.

Desse modo, o objetivo deste estudo foi analisar a densidade básica de 34 espécies arbóreas do Cerradão no estado de Tocantins, e estabelecer a densidade dos troncos em função da densidade dos galhos.

\section{Material e métodos}

\section{Área de estudo}

Amostras de madeira foram coletadas de árvores abatidas em uma área de 10,15 ha de Cerradão no Parque Estadual do Lajeado, localizado em Palmas, TO (10 $\left.10^{\prime} 55^{\prime \prime} \mathrm{S} 48^{\circ} 10^{\prime} 30^{\prime \prime} \mathrm{W}\right)$. O clima predominante na região é do tipo $\mathrm{C} 2 \mathrm{wA}$ 'a', úmido e subúmido com moderada deficiência hídrica no inverno, caracterizado pela ocorrência de duas estações, sendo uma estação seca de maio a setembro e uma estação chuvosa de outubro a abril (Alvares et al., 2014).

\section{Procedimentos metodológicos}

Foram abatidos 80 indivíduos pertencentes a 34 espécies arbóreas do cerrado. O número de indivíduos abatidos para cada espécie (Tabela 1) foi determinado a partir do inventário florestal, onde foi definido que aproximadamente $3 \%$ das árvores com DAP $>5 \mathrm{~cm}$, ou pelo menos um indivíduo de cada espécie seria coletado, mediante autorização da Agência Ambiental do Estado do Tocantins - NATURATINS (Miguel, 2014). De cada indivíduo abatido foram coletadas amostras na forma de discos com $5 \mathrm{~cm}$ de espessura, em três posições do tronco (base, meio e topo) e em três diâmetros dos galhos (galho grosso: diâmetro maior que $10 \mathrm{~cm}$; galho médio: de 3 a $10 \mathrm{~cm}$; e galho fino: menor que $3 \mathrm{~cm}$ ).

Estas amostras do tronco e galho, na condição verde, foram pesadas imediatamente após o abate em balança analítica (sensibilidade de $0,01 \mathrm{~g}$ ), em seguida colocadas em estufa a $103 \pm 2{ }^{\circ} \mathrm{C}$ até massa constante, obtendo a massa anidra a $0 \%$ de umidade. De posse dos dados úmidos e secos, determinou-se o teor de umidade máximo e a densidade básica. $\mathrm{O}$ teor da umidade da madeira (TU) foi calculado segundo norma NBR 7190 (Associação Brasileira de Normas Técnicas, 1997) em função da relação entre a massa úmida $(\mathrm{Mu})$ e a massa seca a $0 \%$ de umidade, conforme equação 1 . A densidade básica $\left(\mathrm{D}_{\mathrm{b}}\right)$ foi determinada pelo método do máximo teor de umidade (Smith, 1954; Vital, 1984), descrito na norma NBR 11941 (Associação Brasileira de Normas Técnicas, 2003), conforme equação 02. 
Tabela 1. Listagem das famílias com respectivas espécies e número de indivíduos arbóreos do Cerradão do estado de Tocantins.

\begin{tabular}{|c|c|c|c|}
\hline $\mathbf{N}^{\circ}$ & Família & Espécie & $\begin{array}{c}\mathrm{N}^{\circ} \text { de } \\
\text { Indivíduos }\end{array}$ \\
\hline 1 & Apocynaceae & Aspidosperma subincanum Mart. & 1 \\
\hline 2 & Malpighiaceae & Byrsonima laxiflora Griseb. & 1 \\
\hline 3 & Malpighiaceae & Byrsonima pachyphylla A. Juss & 1 \\
\hline 4 & Malpighiaceae & Byrsonima sericea A. Juss. $B$ & 2 \\
\hline 5 & Annonaceae & Bocageopsis multiflora (Mart.) & 1 \\
\hline 6 & Fabaceae & Bowdichia virgiloides Kunth. & 1 \\
\hline 7 & Connaraceae & Connarus perrottetti (DC.) Planch & 1 \\
\hline 8 & Connaraceae & Connarus suberosus Planch & 1 \\
\hline 9 & Fabaceae & Dalbergia densiflora Benth. & 1 \\
\hline 10 & Icacinaceae & Emmotum nitens (Benth.) Miers. & 7 \\
\hline 11 & Rubiaceae & $\begin{array}{l}\text { Ferdinandusa elliptica Pohl. Pl. } \\
\text { Bras. }\end{array}$ & 1 \\
\hline 12 & Erythroxylaceae & Erythroxylum daphnites Mart. & 1 \\
\hline 13 & Apocynaceae & $\begin{array}{l}\text { Himatanthus sucuuba (Spruce ex } \\
\text { Mull) Arg.) }\end{array}$ & 1 \\
\hline 14 & Fabaceae & Inga alba (Sw.) Willd. & 1 \\
\hline 15 & Chrysobalanaceae & Licania apetala (E. Meyer) Fritsch. & 1 \\
\hline 16 & Euphorbiaceae & Mabea fistulifera Mart. & 1 \\
\hline 17 & Euphorbiaceae & Maprounea guianensis Aubl. & 2 \\
\hline 18 & Sapindaceae & Matayba guianensis Aubl. & 1 \\
\hline 19 & Lauraceae & Mezilaurus itauba (Meissn.) Taub. & 2 \\
\hline 20 & Melastomataceae & Miconia albicans (Swartz) & 5 \\
\hline 21 & Melastomataceae & Miconia cuspidata Mart. Ex Naudin. & 3 \\
\hline 22 & Myrtaceae & Myrcia splendens (Sw.) DC. & 8 \\
\hline 23 & Ochnaceae & Ouratea ovallis (Pohl) Engl. & 2 \\
\hline 24 & Fabaceae & Parkia pendula (Willd.) Benth. & 2 \\
\hline 25 & Fabaceae & Parkia platycephala Benth. & 1 \\
\hline 26 & Burseraceae & Protium heptaphyllum Mart. & 4 \\
\hline 27 & Vochysiaceae & Qualea parviflora Mart. & 2 \\
\hline 28 & Humiriaceae & Sacoglottis guianensis Benth. & 2 \\
\hline 29 & Sapotaceae & Pouteria ramiflora (Mart.) Radlk. & 5 \\
\hline 30 & Fabaceae & $\begin{array}{l}\text { Tachigali vulgaris L. G. Silva \& H. } \\
\text { C. Lima. }\end{array}$ & 4 \\
\hline 31 & Anacardiaceae & Tapirira guianensis Aubl. & 4 \\
\hline 32 & Myristicaceae & Virola sebifera Aubl. & 2 \\
\hline 33 & Vochysiaceae & Vochysia gardineri Warm. & 1 \\
\hline 34 & Annonaceae & Xylopia aromática (Lam.) Mart. & 7 \\
\hline
\end{tabular}

$$
T U=\frac{M_{U}-M_{0 \%}}{M_{0 \%}} 100
$$

Onde:

TU - Teor de umidade da madeira

$\mathrm{M}_{\mathrm{U}}$ - Massa úmida

$\mathrm{M}_{0 \%}$ - Massa anidra

$$
D_{b}=\frac{1}{\frac{M_{S A T}}{M_{0 \%}}-0,346}
$$

Onde:

$\mathrm{D}_{\mathrm{b}}$ - Densidade básica da madeira

$\mathrm{M}_{\mathrm{SAT}}$ - Massa saturada (massa úmida da amostra, em gramas).

$\mathrm{M}_{0 \%}$ - Massa anidra (massa seca em estufa, em gramas).

A densidade da madeira foi classificada, de acordo com os estudos de Melo et al. (1990); Vale et al. (2002, 2005); Coradin et al. (2010); Silveira et al. (2013), onde se classifica madeiras de baixa densidade aquelas que apresentam valores abaixo de $0,550 \mathrm{~g} \mathrm{~cm}^{-3}$, média densidade aquelas com densidade da madeira entre 0,550 e $0,720 \mathrm{~g} \mathrm{~cm}^{-3}$, e madeiras pesadas ou de alta densidade aquelas com valores superiores a $0,730 \mathrm{~g} \mathrm{~cm}^{-3}$.

Utilizou-se análise de variância (ANOVA), e análise de regressão simples (pacote Stats), implementado na linguagem R (R Development Core Team, 2011). A variável densidade básica de madeira de tronco foi testada para os tratamentos base, meio e topo e também para os tratamentos galho grosso, médio e fino. Para a estimativa da densidade básica do tronco em função da densidade básica do galho utilizou-se a análise de regressão, para dois cenários. No primeiro, foram consideradas todas as árvores de todas as famílias e espécies encontradas no local, considerando as 34 espécies como repetição (espécies com uma árvore foram consideradas com uma repetição, espécies com mais de uma a repetição foi considerada como a média entre elas). No segunda abordagem, foram consideradas apenas aquelas espécies que apresentaram mais de três árvores e estas foram consideradas como repetição, a saber: Emmotum nitens (7), Miconia albicans (5), Myrcia splendens (8), Protium heptaphyllum (4), Pouteria ramiflora (5), Tachigali vulgaris (4) e Tapirira guianensis (4), as quais dominam a área estudada. 
A escolha da equação, que melhor explica a estimativa da densidade básica da madeira do tronco da árvore em função da densidade básica da madeira dos galhos, seguiu os métodos tradicionais utilizados na verificação da regressão na sequência de importância, de acordo Draper \& Smith (1981): análise gráfica dos resíduos (\%) e comportamento do modelo ajustado, em relação à densidade básica real; Erro-padrão da estimativa absoluto e em percentagem $\left(\mathrm{S}_{\mathrm{yx}} ; \mathrm{S}_{\mathrm{yx}}\right)$; Coeficiente de determinação ajustado ( $\mathrm{R}^{2}$ aj.); e valor " $F$ " de Fischer.

Para isso, foi proposta a equação 3 :

$$
D_{B M T}=\beta 0+\beta 1^{*} D_{B M G}
$$

Onde:

$\mathrm{D}_{\mathrm{BMT}}=$ Densidade básica média do tronco $\left(\mathrm{kg} \mathrm{m}^{-3}\right)$

$\beta 0+\beta 1=$ Coeficientes a serem ajustados

$\mathrm{D}_{\mathrm{BMG}}=$ densidade básica média do galho $\left(\mathrm{kg} \mathrm{m}^{-3}\right)$

\section{Resultados e discussão}

As espécies estudadas apresentaram $0,650 \mathrm{~g} \mathrm{~cm}^{-3}$ de valor médio para a densidade básica da madeira para o povoamento (Figura 1). Entre as espécies, 6 apresentaram baixa densidade, 21 espécies apresentaram média densidade, englobando cerca de $61 \%$ das espécies arbóreas analisadas neste estudo, e 7 espécies podem ser classificadas como madeira pesada. Se destacam entre as espécies com densidades mais altas Miconia cuspidata $\left(0,845 \mathrm{~g} \mathrm{~cm}^{-3}\right)$, Emmotum nitens $(0,841$ $\left.\mathrm{g} \mathrm{cm}^{-3}\right)$ e Bowdichia virgiloides $\left(0,827 \mathrm{~g} \mathrm{~cm}^{-3}\right)$. As espécies de menor densidade foram Connarus perrottetti $\left(0,453 \mathrm{~g} \mathrm{~cm}^{-3}\right)$ e Vochysia gardineri $\left(0,352 \mathrm{~g} \mathrm{~cm}^{-3}\right)$.

A densidade básica da madeira do tronco para a maioria das espécies (58\%) decresceu da base para o topo. Como exemplo, destaca-se Sacoglottis guianensis, cujo padrão é o mais comum entre as espécies amostradas. Do restante das espécies, $15 \%$ apresentaram densidades iguais na base e no meio do tronco, diminuindo para o topo, como observado em Aspidosperma subincanum. Outras $27 \%$ apresentaram comportamento de queda da densidade da base para o meio do fuste, permanecendo constante, a partir daí até o topo, como em Myrsia splendens (Figura 2).

Ao analisar a madeira do galho observou-se que a maioria das espécies (88\%) apresentou densidade básica maior para os galhos grossos, seguido de galhos médios e finos, como registrado para Emmotum nitens (Figura 3). A densidade básica da madeira dos galhos variou entre $0,327 \mathrm{~g} \mathrm{~cm}^{-3}$ em galhos finos de Vochysia gardineri e $0,836 \mathrm{~g} \mathrm{~cm}^{-3} \mathrm{em}$ galhos grossos de Bowdichia virgiloides. Apenas duas espécies (6\%) apresentaram densidade igual para galhos grossos e médios, aumentando nos galhos finos, como registrado para Inga alba. Contudo, outras duas espécies $(6 \%)$ apresentaram maior densidade para galhos médios, a exemplo de Licania apetala (Figura 3).

A densidade básica da madeira das espécies estudadas encontra-se dentro do esperado para espécies tropicais. A exemplo, o estudo de Jati et al. (2014), com espécies da savana de Roraima no norte da Amazônia brasileira, registrou densidade básica da madeira de $0,592 \mathrm{~g} \mathrm{~cm}^{-3}$ (média geral). Valores de densidade básica semelhantes aos registrados nesta pesquisa foram relatados com espécies tropicais nos estudos de Baker et al. (2004); Bucci et al. (2004); Quirino et al. (2005); Chave et al. (2006); Barcellos (2007); Goulart et al. (2012) e Oliveira et al. (2012a). Por outro lado, Vale et al. (2002) registraram valores da densidade básica da madeira de espécies do Cerrado, variando de $0,20 \mathrm{~g} \mathrm{~cm}^{-3}$ a $0,78 \mathrm{~g} \mathrm{~cm}^{-3} \mathrm{e}$ Cintra (2009) encontrou valores entre 0,316 e $0,786 \mathrm{~g} \mathrm{~cm}^{-3}$.

Em estudo onde foi analisada a densidade básica em diferentes fitofisionomias do Cerrado em Minas Gerais, Oliveira et al. (2012b) observaram que a variação da densidade pode ser explicada pela variação de fatores edáficos, topográficos, clima sazonal, condições hídricas, fenologia e forma de dispersão das espécies e a incidência de fogo. Além desses, Barcellos (2007) relata que fatores genéticos, ambientais e fisiográficos também podem influenciar na massa específica básica da madeira.

Dentro de uma mesma árvore também ocorre variação da densidade da madeira, assim como entre indivíduos de uma mesma espécie, tal variabilidade ocorre em função de fatores genéticos (gêneros, espécies, procedências, etc.), fatores do meio (clima, solo, topografia, etc.) e silviculturais (espaçamento, fertilização, idade de corte, desbaste, desrama, etc. (Vale et al., 1999). 


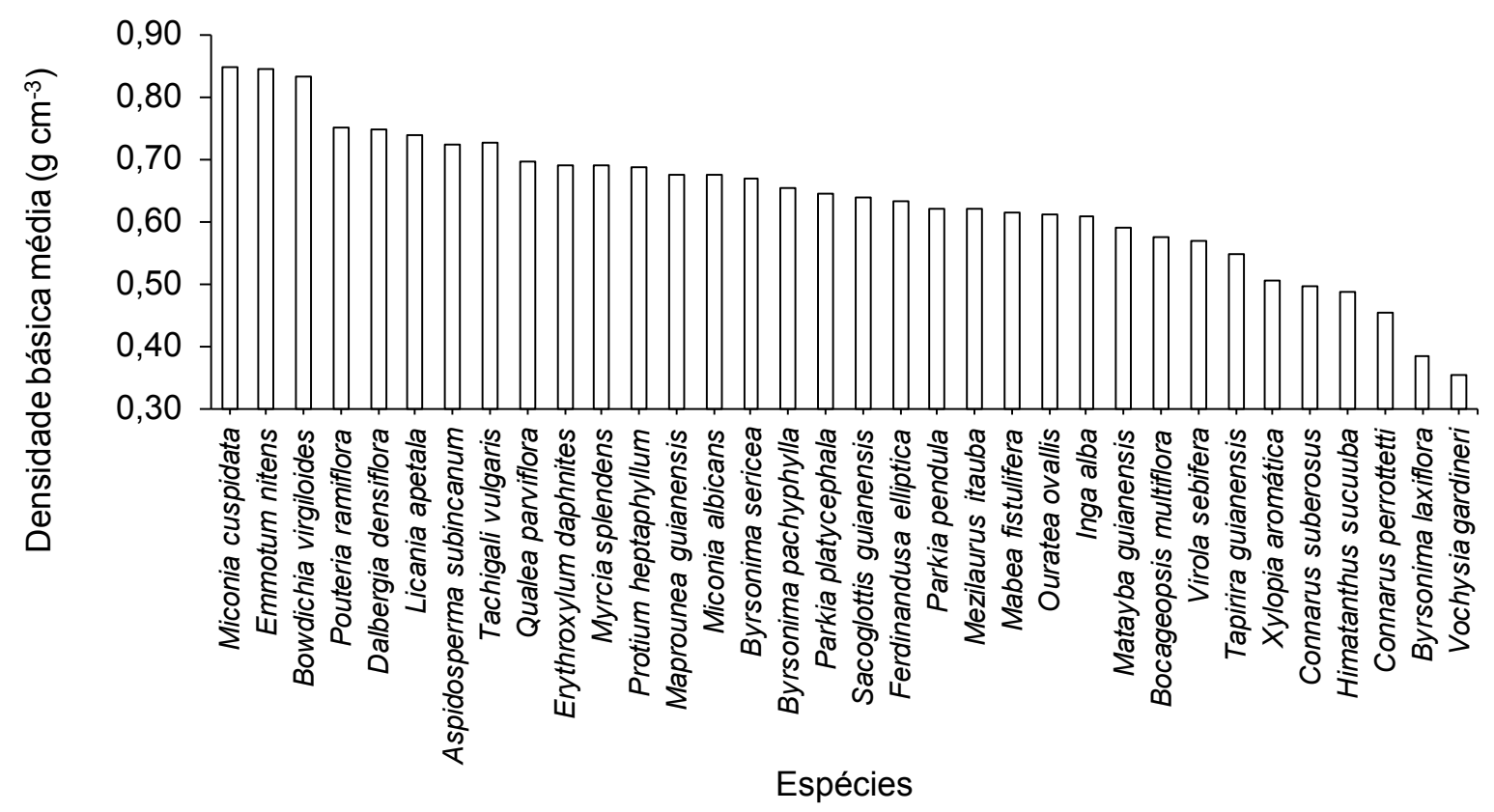

Figura 1. Densidade básica média de 34 espécies arbóreas de Cerradão, em Palmas, TO.

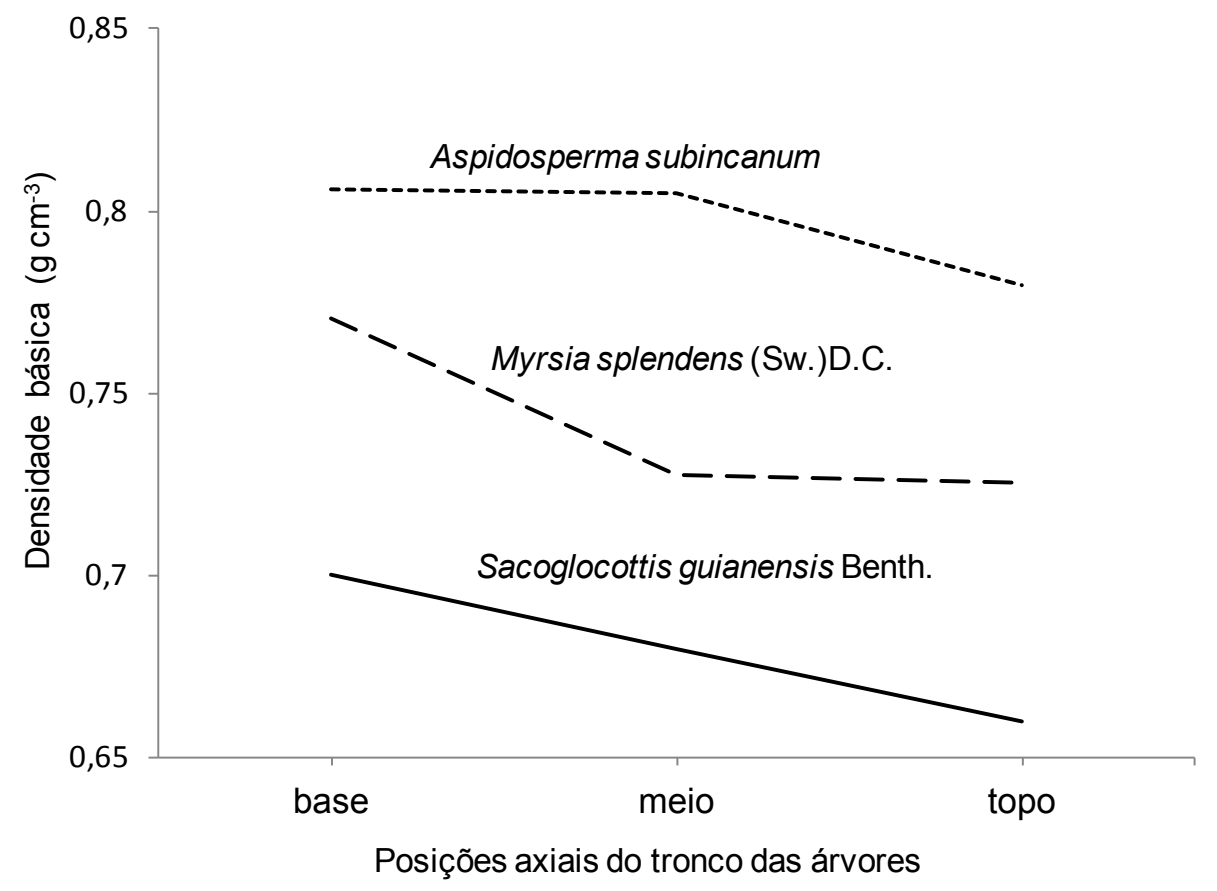

Figura 2. Variação axial da densidade básica de madeira de tronco em árvores de Cerradão, em Palmas, TO. 


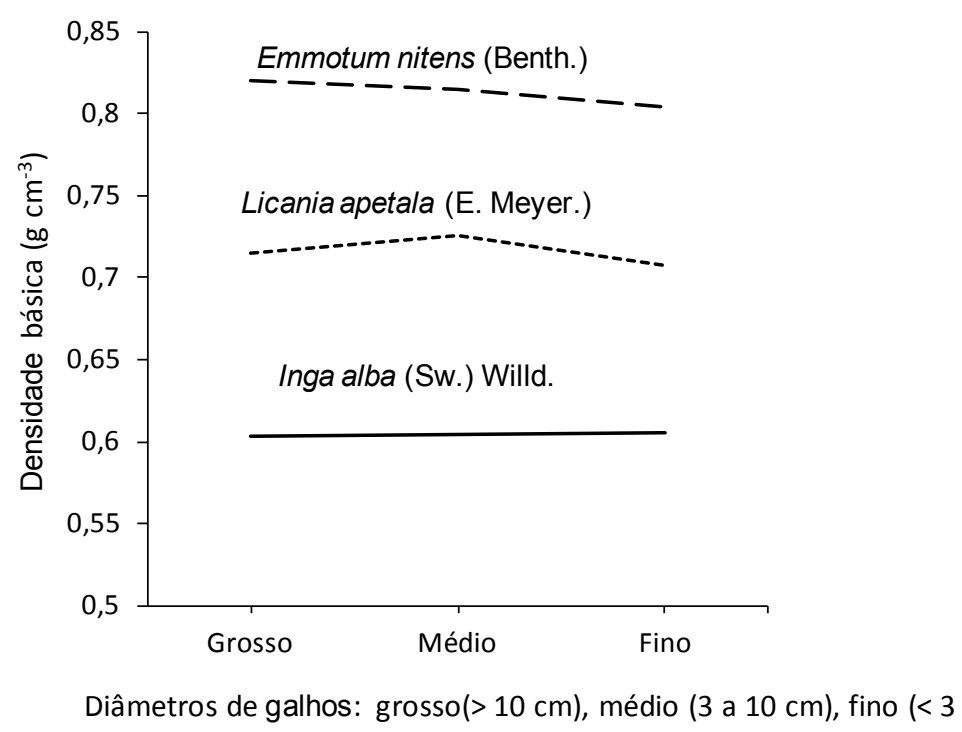

Figura 3. Densidade da madeira de galhos grosso, médio e fino para espécies de Cerradão, em Palmas, TO.

A densidade básica influencia a velocidade de queima da madeira durante a produção de energia (Cintra, 2009). Logo, madeiras de maior densidade apresentam maior potencial para produção de carvão vegetal, pois dão origem a produtos com maior poder calorífico (Santos, 2010). Contudo, estudos com madeiras nativas são necessários para que se possam ter indicativos do potencial de uso dessas espécies para finalidades energéticas, direcionando, assim, avanços nas pesquisas de melhoramento genético (Costa et al., 2014).

A densidade básica da madeira entre as partes do tronco e dos galhos apresentaram relação significativa para as espécies analisadas (Tabela 2 e Figura 4), exceto para o galho grosso, que não apresenta relação significativa com as densidades básicas dos galhos fino e médio $\left(\mathrm{R}^{2}=0,2 ; \mathrm{p}<0,003\right)$. De forma semelhante, foi registrada relação significativa entre partes do tronco e partes do galho (Tabela 3 e Figura 5), exceto a densidade do galho grosso que também não apresentou relação significativa com as partes do tronco $\left(\mathrm{R}^{2}=0,2\right.$; $\mathrm{p}<0,006)$. Isso indica que espécies que apresentaram alta densidade da madeira no tronco também apresentarão alta densidade da madeira nos galhos.
Tabela 2. Resultado da relação da densidade da madeira entre partes do tronco e dos galhos de espécies arbóreas de Cerradão, em Palmas, TO.

\begin{tabular}{lrrll}
\hline \multicolumn{1}{c}{ Variáveis } & $\mathbf{R}^{2}$ & $\mathbf{F}$ & \multicolumn{1}{c}{$\mathbf{p}$} & \multicolumn{1}{c}{ Resíduo } \\
\hline Tronco (Base x Meio) & 0,9903 & 3.253 & $2,2^{-16}$ & 0,01208 \\
Tronco (Base x Topo) & 0,9788 & 1.475 & $2,2^{-16}$ & 0,01785 \\
Tronco (Meio x Topo) & 0,9897 & 3.082 & $2,2^{-16}$ & 0,01193 \\
Galho (Grosso x Médio) & 0,241 & 10,16 & 0,003200 & 0,1767 \\
Galho (Grosso x Fino) & 0,2526 & 10,81 & 0,002452 & 0,1753 \\
Galho (Médio x Fino) & 0,9797 & 1.546 & $2,2^{-16}$ & 0,0158 \\
\hline
\end{tabular}

Onde: $\mathrm{R}^{2}=$ Coeficiente de determinação amostral; $\mathrm{F}=$ Frequência absoluta acumulada, $\mathrm{p}=$. Nível crítico amostral (significância).

Tabela 3. Resultado da relação da densidade da madeira entre partes do tronco dos galhos de espécies arbóreas de Cerradão, em Palmas, TO.

\begin{tabular}{lllll}
\hline \multicolumn{1}{c}{ Variáveis } & \multicolumn{1}{c}{$\mathbf{R}^{\mathbf{2}}$} & \multicolumn{1}{c}{$\mathbf{F}$} & \multicolumn{1}{c}{$\mathbf{p}$} & \multicolumn{1}{c}{ Resíduo } \\
\hline Tronco base x galho grosso & 0,224 & 9,239 & 0,004693 & 0,1079 \\
Tronco meio x galho grosso & 0,2176 & 8,901 & 0,005418 & 0,1041 \\
Tronco topo x galho grosso & 0,2545 & 10,93 & 0,002345 & 0,0994 \\
Tronco base x galho médio & 0,8695 & 213,3 & $1,052^{-15}$ & 0,04424 \\
Tronco meio x galho médio & 0,8984 & 282,8 & $2,2^{-16}$ & 0,03753 \\
Tronco topo x galho médio & 0,9222 & 379,3 & $2,2^{-16}$ & 0,03211 \\
Tronco base x galho fino & 0,8351 & 162,1 & $4,536^{-14}$ & 0,04973 \\
Tronco meio x galho fino & 0,8618 & 199,6 & $2,646^{-15}$ & 0,04376 \\
Tronco topo x galho fino & 0,8872 & 251,8 & $2,2^{-16}$ & 0,03866 \\
\hline
\end{tabular}

Onde: $\mathrm{R}^{2}=$ Coeficiente de determinação amostral; $\mathrm{F}=$ Frequência absoluta acumulada, $\mathrm{p}=$. Nível crítico amostral (significância). 

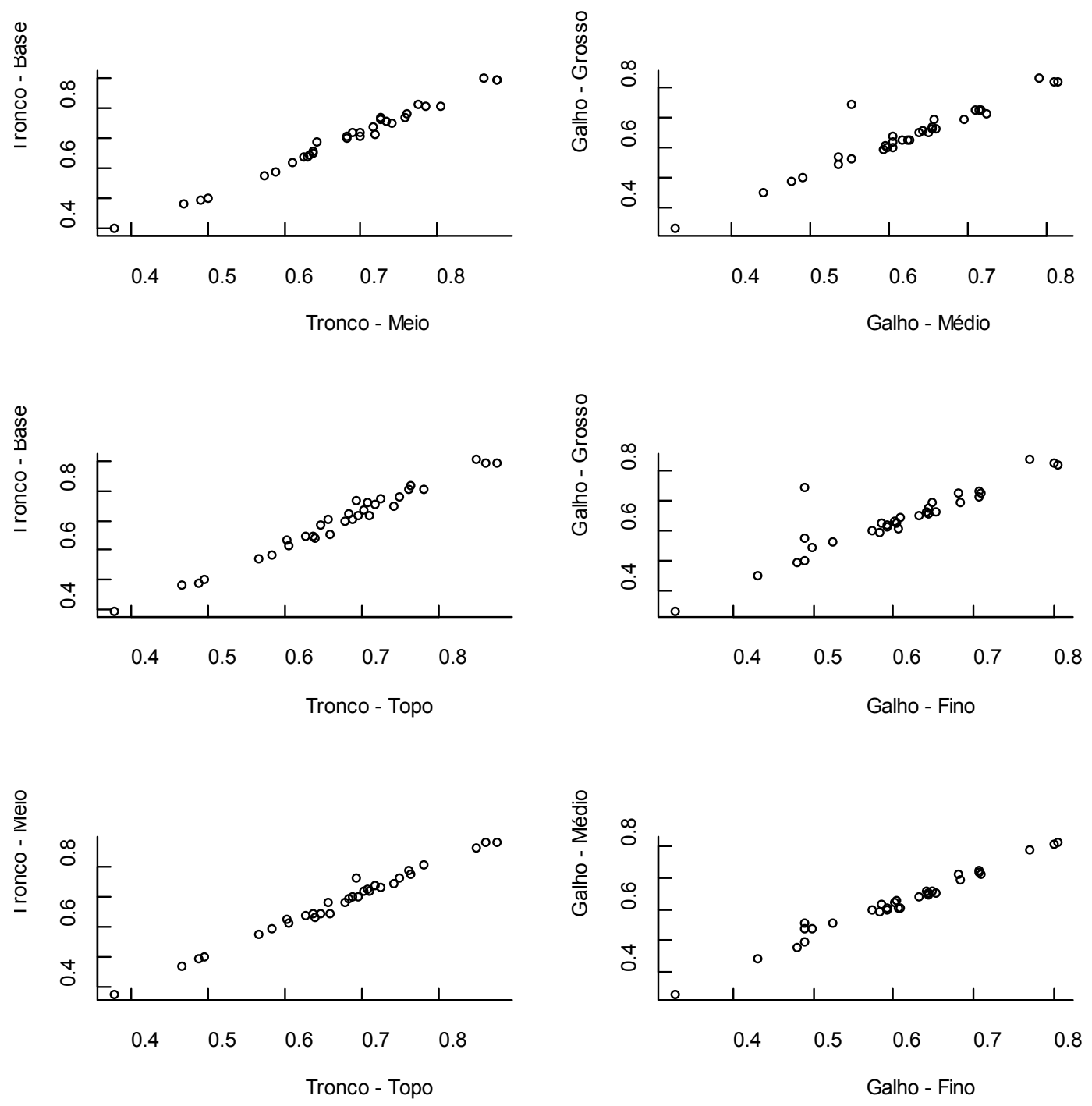

Figura 4. Relação da densidade $\left(\mathrm{g} \mathrm{cm}^{-3}\right)$, entre partes do tronco e dos galho de espécies arbóreas de Cerradão, em Palmas, TO. Foram consideradas espécies com mais de 3 indivíduos amostrados.

De acordo com os modelos propostos para estimar densidade do tronco em função da densidade de galhos grossos, médios e finos, a variável densidade do galho médio (diâmetro de $3-10 \mathrm{~cm}$ ) apresentou o melhor ajuste na equação, em seguida a densidade do galho fino e galho grosso (Tabela 4). Observando as Figuras 6, 7, 8, e 9, é possível verificar essa relação entre a densidade básica do tronco e as densidades: galhos finos, galhos médios, galhos grossos e a média dos galhos. A distribuição dos resíduos não ultrapassou uma variação de $20 \%$ tanto para subestimar ou superestimar a densidade básica da árvore pela média dos galhos das 34 espécies. A mesma análise foi feita para os galhos finos, médios e grossos separadamente onde os galhos finos apresentaram uma variação de $15 \%$, os galhos médios uma variação de $20 \%$ para subestimar ou superestimar a densidade básica da árvore. No entanto, quando se utilizou a densidade básica dos galhos grossos essa variação foi de $10 \%$ para subestimar e de $90 \%$ para superestimar a densidade básica da árvore. Baseado nesses resultados pode-se obter a densidade da árvore utilizando galhos médios, finos e grossos sendo os galhos médios com diâmetro entre 3 e $10 \mathrm{~cm}$ os mais indicados. 

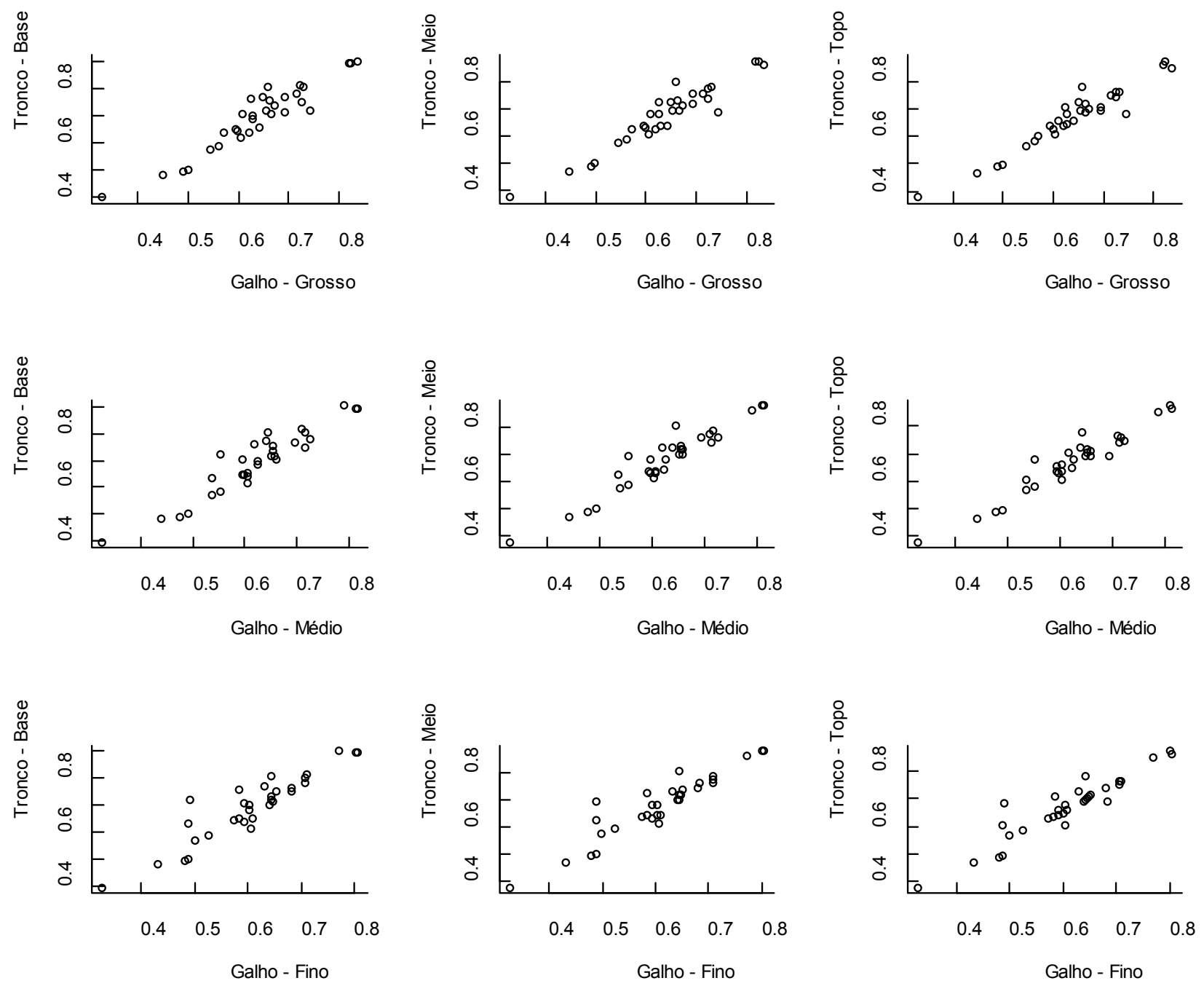

Figura 5. Relação da densidade $\left(\mathrm{g} \mathrm{cm}^{-3)}\right.$ entre partes do tronco e dos galhos de espécie arbóreas de Cerradão em Palmas, TO. Foram consideradas espécies com mais de 3 indivíduos amostrados. 
Tabela 4. Ajuste do modelo para estimar a densidade do tronco (DT) em função da densidade dos galhos de 34 espécies arbóreas de Cerradão, em Palmas, TO.

\begin{tabular}{lccccc}
\hline Galho & $\begin{array}{c}\text { Modelo } \\
D_{B M T}=\beta 0+\beta 1^{*} D_{B M G}\end{array}$ & $\mathbf{R}^{2}$ aj. & Syx & $\begin{array}{c}\text { Syx } \\
(\mathbf{\%})\end{array}$ & F \\
\hline Fino & $\mathrm{D}_{\mathrm{BMT}}=0,098346813+0,954203851 * \mathrm{D}_{\mathrm{BMGF}}$ & 0,88 & 0,035 & 5,16 & 608,7382 \\
Médio & $\mathrm{D}_{\mathrm{BMT}}=0,061581+0,988638 * \mathrm{D}_{\mathrm{BMGM}}$ & 0,90 & 0,033 & 4,76 & 727,408 \\
Grosso & $\mathrm{D}_{\mathrm{BMT}}=0,03892+1,003672 * \mathrm{D}_{\mathrm{BMGG}}$ & 0,89 & 0,033 & 4,79 & 651,6024 \\
Média & $\mathrm{D}_{\mathrm{BMT}}=0,059507979+0,994111508 * \mathrm{D}_{\mathrm{MG}}$ & 0,91 & 0,030 & 4,41 & 861,625 \\
\hline
\end{tabular}

Onde: $\beta$ 's = Coeficientes estimados; $\mathrm{R}^{2} \mathrm{Aj}=$ Coeficiente de determinação Syx e Syx $\%$ = Erro padrão da estimativa e erro padrão da estimativa em percentagem; $\mathrm{F}=$ Valor de $\mathrm{F}$ da análise de variância, $\mathrm{DT}=$ densidade do tronco; $\mathrm{DG}=$ densidade do galho ( $\mathrm{F}=$ fino, $\mathrm{M}=$ médio, $\mathrm{G}=$ grosso, $\mathrm{MG}=$ média dos galhos).

A

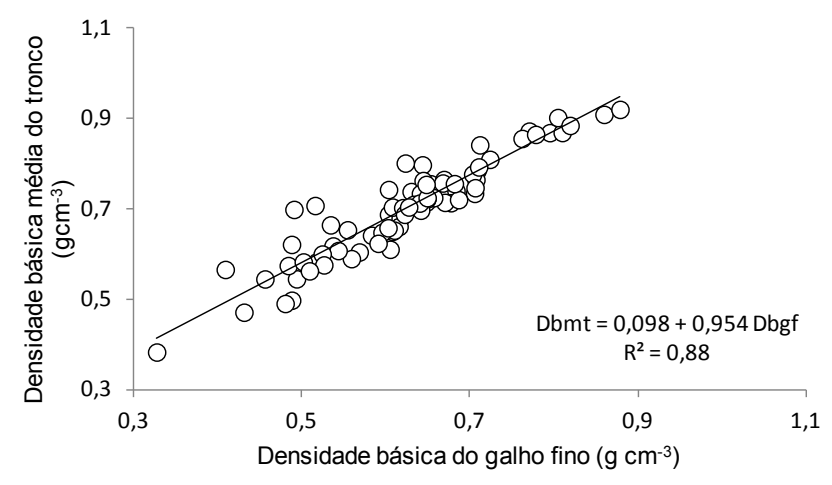

B

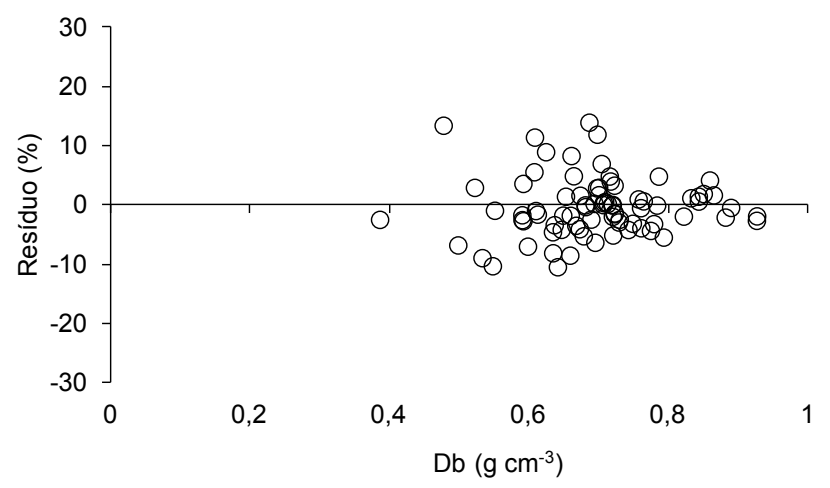

Figura 6. Densidade básica média da madeira do tronco em função da densidade básica do galho fino $(<3 \mathrm{~cm})$ para as 34 espécies lenhosas de Cerradão, em Palmas, TO (A) e distribuição dos resíduos (B). 
A

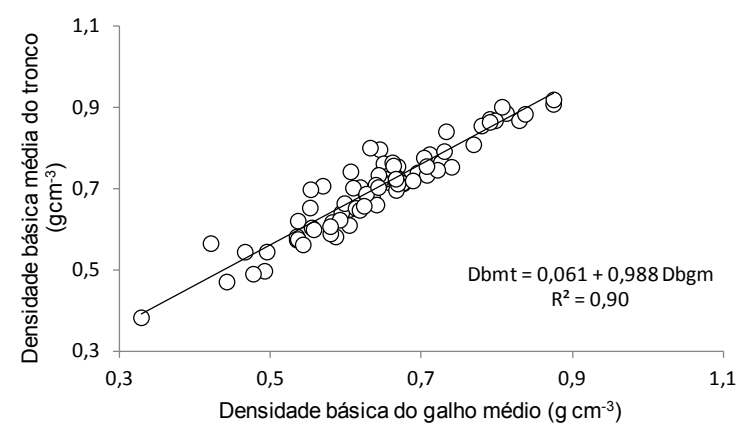

B

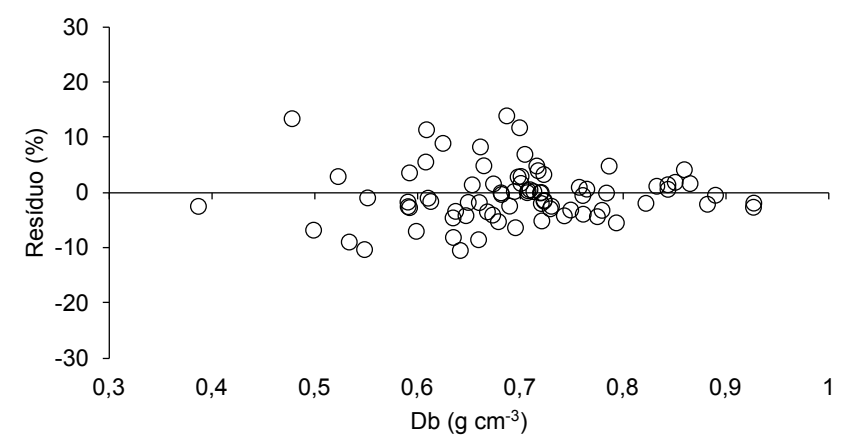

Figura 7. Densidade básica média da madeira do tronco em função da densidade básica do galho médio (3 a $10 \mathrm{~cm})$ para as 34 espécies lenhosas de Cerradão, em Palmas, TO (A) e distribuição dos resíduos (B).

A

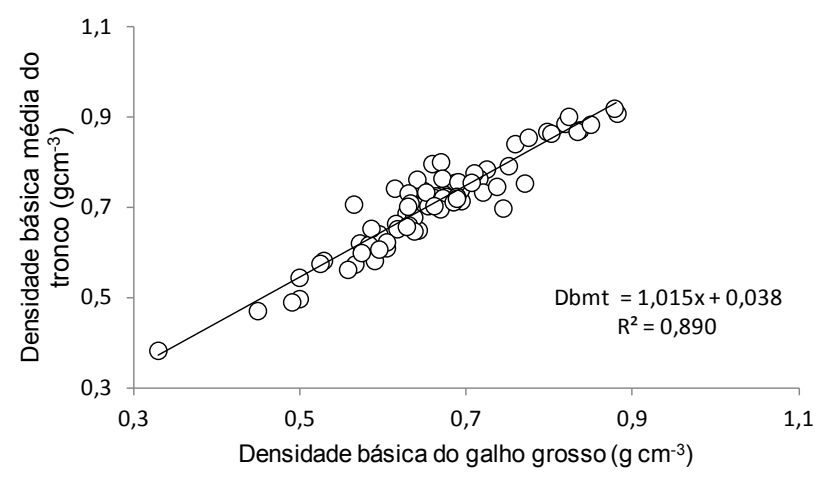

B

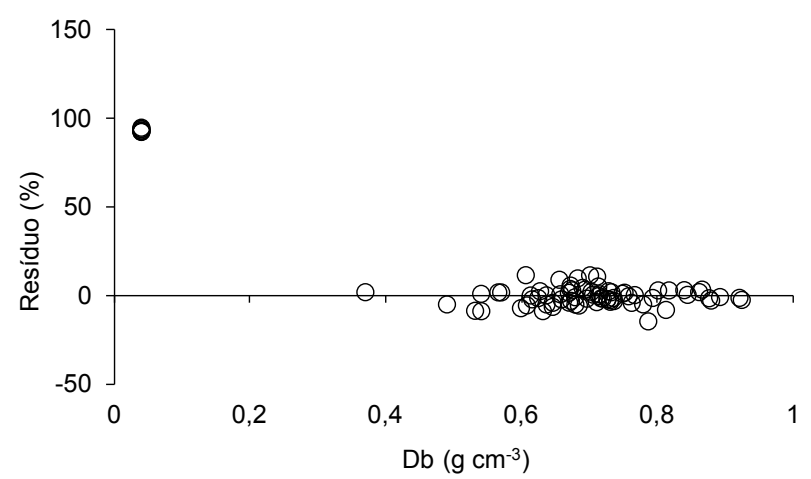

Figura 8. Densidade básica média da madeira do tronco em função da densidade básica do galho grosso $(>10 \mathrm{~cm})$ para as 34 espécies lenhosas de Cerradão, em Palmas, TO (A) e distribuição dos resíduos (B).

A

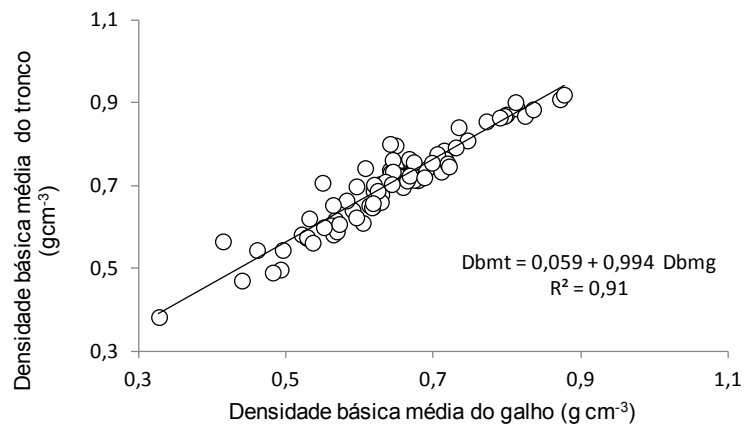

B

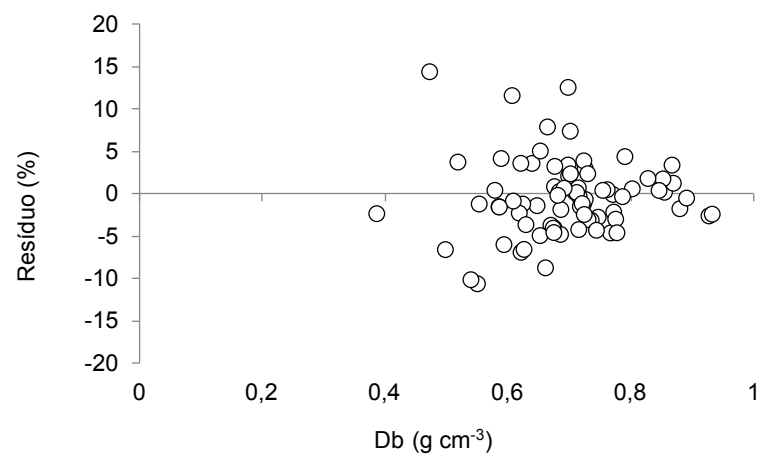

Figura 9. Densidade básica média da madeira do tronco em função da densidade básica média dos galhos para as 34 espécies lenhosas de Cerradão, em Palmas, TO (A) e distribuição dos resíduos (B). 
Os resultados obtidos neste estudo mostram que é possível estimar a densidade da madeira do tronco de uma árvore do Cerradão a partir de amostras da madeira de galhos finos, médios e grossos, mesmo que os galhos apresentem menor densidade da madeira que o tronco. $\mathrm{O}$ coeficiente de determinação $\left(\mathrm{R}^{2}\right)$ demonstrou que $88 \%$ da variação da densidade registrada no tronco pode ser explicada pela densidade dos galhos, com erros médios percentuais inferiores a 5\%, conforme o erro padrão da estimativa (Syx\%). Além disso, foi registrada relação linear positiva $\left(\mathrm{R}^{2}>0,94\right)$ entre a densidade do fuste e a dos galhos (Figura 10). Logo, por meio da matriz de correlação de Scatterplots, é possível constatar que a densidade dos galhos aumenta com a densidade do tronco.

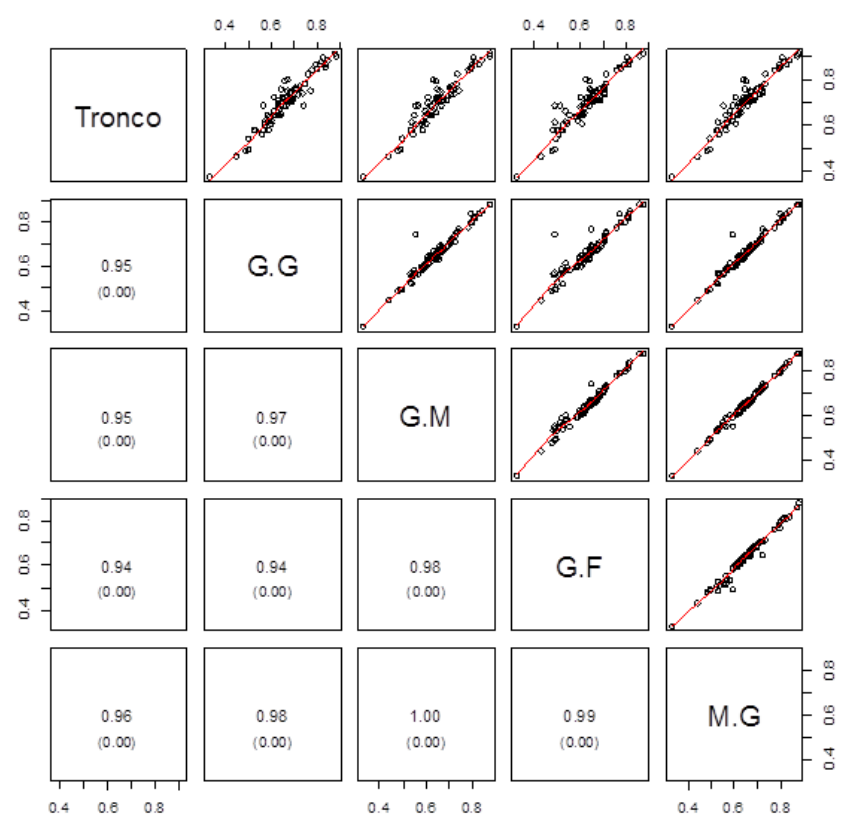

Figura 10. Matriz de correlação de Scaterplots para as variáveis galho fino (G.F), galho médio (G.M), galho grosso (G.G) e média dos galhos (M.G) em função da variável densidade do tronco.

Densidade da madeira de galhos e troncos diferente e tendência de aumento da densidade no sentido do topo para a base da árvore também foram registradas por Jati et al. (2014), nas savanas amazônicas e por Valério et al. (2008), no estudo de densidade básica de Cedrela fissilis Vell. Além disso, a ausência de diferença significativa entre as posições do galho e do tronco registrada neste estudo foi também corroborada pelos dados de Silveira et al. (2013), que avaliaram a densidade básica de três espécies Amazônicas. Portanto, sugere-se a caracterização da densidade básica da madeira de um indivíduo arbóreo a partir da análise dos galhos. Jati et al. (2014) veem como vantajosa a coleta de peças dos galhos com diâmetro entre 5 e $10 \mathrm{~cm}$ para estimar, através de regressão, a densidade da madeira do indivíduo arbóreo com base do tronco $>10 \mathrm{~cm}$. Isso evita o uso do método destrutivo dos indivíduos e pode reduzir erros de superestimativas nos estoques de biomassa/carbono arbóreo, devido ao uso tradicional de amostras derivadas do tronco.

\section{Conclusões}

O padrão de densidade da madeira dos galhos de baixa, média ou alta densidade, foi o mesmo registrado nos troncos para 34 espécies de Cerradão de Palmas, TO, sendo possível estimar a densidade do tronco da árvore utilizando amostras dos galhos. Os galhos médios (diâmetro entre 3 e $10 \mathrm{~cm}$ ) são os mais indicados para este fim, pois apresentaram o melhor ajuste no modelo proposto. Os métodos usados neste estudo foram adequados para melhorar as estimativas de estoque de carbono arbóreo em áreas de cerrado, evitando-se amostragem destrutiva. Os resultados obtidos com a análise de um indivíduo por espécie devem ser considerados como um indicativo para confirmações de pesquisas futuras as quais devem fazer amostragens mais abrangentes.

\section{Referências}

ALVARES, C. A.; STAPE, J. L.; SENTELHAS, P. C.; GONÇALVES, J. L. M.; SPAROVEK, G. Koppen's climate classification map for Brazil. Meteorologische Zeitschrift, Berlin, v. 22, n. 6, p. 711-728, 2014. DOI: $10.1127 / 0941-2948 / 2013 / 0507$

ASSOCIAÇÃO BRASILEIRA DE NORMAS TÉCNICAS. NBR 7190: projeto de estruturas de madeira. Rio de Janeiro, 1997. 107 p. ASSOCIAÇÃO BRASILEIRA DE NORMAS TÉCNICAS. NBR 11941: madeira: determinação da densidade básica. Rio de Janeiro, 2003. 6 p.

BAKER, T. R.; PHILLIPS, O. L.; MALHI, Y.; ALMEIDA, S.; ARROYO, L.; DIFIORE, A. T. E.; LAURANCE, S. G.; LAURANCE, W. F. LEWIS, S. L.; LLOYD, J.; MONTEAGUDO, A.; NEIL, L. D. A.; PAT I N, S.; PITMAN N. G. E. A. J.; SILVA, N. M.; MARTINS, R. V. Variation in wood density determines spatial patterns in Amazonian forest biomass. Global Change Biology, Oxford, v. 10, p. 545-562, 2004.

Pesq. flor. bras., Colombo, v. 35, n. 82, p. 63-75, abr.jun. 2015 
BARCELLOS, D. C. Caracterização do carvão vegetal através do uso de espectroscopia no infravermelho próximo. 2007. 140 f. Tese (Doutorado em Scientiae) - Universidade Federal de Viçosa, Viçosa, MG.

BOWYER, J. L.; SHMULSKY, R.; HAYGREEN, J. G. Forest products and wood science: an introduction. New York: Blackwell Publishing, 2003.

BUCCI, S. J.; GOLDSTEIN, G.; MEINZER, F. C.; SCHOLZ, F. G.; FRANCO, A. C.; BUSTAMANTE, M. Functional convergence in hydraulic architecture and water relations of tropical savanna trees: from leaf to whole plant. Tree Physiology, v. 24, p. 891-899, 2004.

CHAVE, J.; MULLER-LANDAU, H. C.; BAKER, T. R.; EASDALE, T. A.; STEEGE, H.T.; WEBB, C. O. Regional and phylogenetic variation of wood density across 2456 Neotropical tree species. Ecological Applications, Tempe, v. 16, p. 2356-2367, 2006.

CINTRA, T. C. Avaliação energética de espécies florestais nativas plantadas na região do médio Paranapanema, SP. 2009. Dissertação (Mestrado em Recursos Florestais) - Escola Superior de Agricultura Luiz de Queiroz, Universidade de São Paulo, Piracicaba.

CORADIN, V. T. R.; CAMARGOS, J. A. A.; PASTORE, T. C. M.; CHRISTO, A. G. Madeiras comerciais do Brasil: chave interativa de identificação baseada em caracteres gerais e macroscópicos. 2010. Disponível em: <http://www.ibama.gov.br/lpf/madeira/introducao. htm>. Acesso em: 25 ago. 2014.

COSTA, T. G.; BIANCHI, M. L.; PROTÁSIO, T. P.; TRUGILHO, P. F.; PEREIRA, A. J. Qualidade da madeira de cinco espécies de ocorrência no cerrado para produção de carvão vegetal. Cerne, Lavras, v. 20, n. 1, p. 37-46, 2014. DOI: 10.1590/S010477602014000100005

COSTA, V. E. Caracterização físico-energética da madeira e produtividade de reflorestamentos de clones de híbridos de Eucalyptus grandis $\mathbf{x}$ E. urophylla. 2006. 79 f. Tese (Doutorado em Agronomia) - Universidade Estadual Paulista, Botucatu.

DRAPER, N. R.; SMITH, H. Applied regression analysis. New York: J. Wiley, 1981. 709 p.

GOULART, S. L.; MORI, F. A.; RIBEIRO, A. O. COUTO, A. M.; ARANTES, M. D. Ch.; MENDES, L. M. Análises químicas e densidade básica da madeira de raiz, fuste e galho 59 de barbatimão [(Stryphnodendron adstringens $)$ coville] de bioma cerrado. Cerne, Lavras, v. 18, n. 1, p. 59-66, 2012. DOI: 10.1590/S010477602012000100008

JATI, S. R.; FEARNSIDE, P. M.; BARBOSA, R. I. Densidade da madeira de árvores em savanas do norte da Amazônia brasileira. Acta Amazônica, Manaus, v. 44, n. 1, p. 79-86, 2014. DOI: 10.1590/ S0044-59672014000100008

MELO, J. E.; CORADIN, V. R.; MENDES, J. C. Classes de densidade para madeiras da Amazônia brasileira. In: CONGRESSO FLORESTAL BRASILEIRO, 6., 1990, Campos do Jordão. Anais... Campos do Jordão, 1990. v. 3. p. 695-705.

MIGUEL, E. P. Caracterização da flora e da produção em volume, biomassa e carbono da vegetação arbórea em área de cerradão no estado do Tocantins. 2014. 96 f. Tese (Doutorado em Ciências Florestais) - Universidade de Brasília, Brasília, DF.

Pesq. flor. bras., Colombo, v. 35, n. 82, p. 63-75, abr./jun. 2015
MULLER-LANDAU, H. C. Interspecific and inter-site variation in wood specific gravity of tropical trees. Biotropica, Washington, DC, v. 36, p. 20-32, 2004.

OLIVEIRA, J. T. S.; HELLMEISTER, J. C.; TOMAZELLO FILHO, M. Variação do teor de umidade e da densidade básica na madeira de sete espécies de eucalipto. Revista Árvore, Viçosa, MG, v. 29, n. 1, p. 115-127, 2005. DOI: 10.1590/S0100-67622005000100013

OLIVEIRA, A. C.; ROCHA, M. F. V.; PEREIRA, B. L.; CARNEIRO, A. C. O.; CARVALHO, A. M. M. L.; VITAL, B. R. Avaliação de diferentes níveis de desbaste nas propriedades da madeira e do carvão vegetal de Eucalyptus grandis x Eucalyptus urophylla. Floresta, Curitiba, v. 42, n. 1, p. 59-68, 2012a. DOI: 10.5380/rf.v42i1.26297

OLIVEIRA, G. M. V.; MELlO, J. M.; TRUGILHO, P. F.; SCOLFORO, J. R. S.; ALtOÉ, T. F.; SILVA-NETO, A. J.; OLIVEIRA, A. D. Efeito do ambiente sobre a densidade da madeira em diferentes fitofisionomias do estado de Minas Gerais. Cerne, Lavras, v. 18 , p. $345-352$, 2012b. DOI: $10.1590 / \mathrm{S} 0104-$ 77602012000200020

PRESTON, K. A.; CORNWELL, W. K.; DENOYER, J. L. Wood density and vessel traits as distinct correlates of ecological strategy in 51 California coast range angiosperms. New Phytologist, Oxford, v. 170, p. $807-818,2006$.

PRINGLE, L. Ecologia: a ciência da sobrevivência. Rio de Janeiro: Biblioteca do Exército, 1997. 52 p.

QUIRINO, W. F.; VALE, A. T.; ANDRADE, A. P. A.; ABREU, V. L. S.; AZEVEDO, A. C. S. Poder calorífico da madeira e de matérias lignocelulosicos. Revista da Madeira, São Paulo, n. 89, p. 100-106, 2005.

R DEVELOPMENT CORE TEAM. R: a language and environment for statistical computing. Vienna: R Foundation for Statistical Computing, 2011. Disponível em: <http://www.R-project.org/.2011>. Acesso em: 11 fev. 2013.

RIBEIRO, F. A.; ZANI FILHO, J. Variação da densidade básica da madeira em espécies/procedências de Eucalyptus spp. IPEF, Piracicaba, n. 46, p. 76-85, 1993.

SANTOS, R. C. dos. Parâmetros de qualidade da madeira e do carvão vegetal de clones de Eucalipto. 2010. 159 f. Tese (Doutorado em Ciências Florestais) - Universidade Federal de Lavras, Lavras.

SIH, G. C. Strain: energy density factor applied to mixed mode crack problems. International Journal of Fracture, v. 10, n. 3, 1974.

SILVEIRA, L. H. C.; REZENDE, A. V.; VALE, A. T. Teor de umidade e densidade básica da madeira de nove espécies comerciais amazônicas. Acta Amazônica, Manaus, v. 43, n. 2, p.179 - 184, 2013. DOI: $10.1590 / \mathrm{S} 0044-59672013000200007$

SMITH, D. M. Maximum moisture content method for determining specific gravity of small wood samples. Madison: USDA Forest Service, 1954. 8 p. (USDA Forest Service, 2014).

TRUGILHO, P. F.; SILVA, D. A.; FRAZÃO, F. J. L.; MATOS, J. L. M. Comparação de métodos de determinação da densidade básica em madeira. Acta Amazônica, Manaus, v. 20, p. 307-319, 1990.

VALE, A. T.; BRASIL, M. A. M.; LEÃO, A. L. Quantificação e caracterização energética da madeira e casca de espécies do cerrado. Ciência Florestal, Santa Maria, RS, v. 12, n. 1, p. 71-80, 2002. 
VALE, A. T.; BRASIL, M. A. M.; MARTINS, I. S. Variação axial da densidade básica da madeira de Acacia mangium Willd aos sete anos de idade. Ciência Florestal, Santa Maria, RS, v. 9, n. 2, p. 85-92, 1999.

VALE, A. T.; SARMENTO, T. R.; ALMEIDA, A. N. Caracterização e uso de madeiras de galhos de árvores provenientes da arborização de Brasília - DF. Ciência Florestal, Santa Maria, RS, v. 15, n. 4, p. 411-420, 2005.
VALÉRIO, A. F.; WATZLAWICK, L. F.; SILVESTRE, R.; KOEBLER, H. S. Determinação da densidade básica da madeira de cedro (Cedrela fissilis Vell.) ao longo do fuste. Pesquisa Aplicada \& Agrotecnologia, Guarapuava, v. 1, n. 1, 2008.

VITAL, R. B. Métodos de determinação da densidade da madeira. Boletim Técnico, Viçosa, MG, n. 1, p. 21, 1984.

WILliAMSON, G. B.; WIEMANN, M. C. Age versus size determination of radial variation in wood specific gravity: lessons from eccentrics. Trees, v. 25, p. 585-591, 2011. DOI: 10.1007/ s00468-010-0535-5 
